\title{
The Effect of Rapid Thermal Annealing on InAs/GaAs Quantum Dot Solar Cells
}

Phu Minh Lam, Jiang Wu, Sabina Hatch, Dongyoung Kim, Mingchu Tang, Huiyun Liu

Department of Electronic and Electrical Engineering, University College London, Torrington Place, London WC1E 7JE, United Kingdom

James Wilson and Rebecca Allison

Defence Science and Technology Laboratory, Portsdown West, Portsdown Hill road, Fareham Hants PO17 $6 A D$, United Kingdom

*Email: Phu.Lam@ucl.ac.uk

\begin{abstract}
The effect of post-growth annealing on InAs/GaAs quantum dot solar cells (QDSCs) has been studied. A significant improvement in photoemission, photocurrent density, and spectral response has been observed with post-growth annealing. The optimal anneal temperature was found to be $700^{\circ} \mathrm{C}$, which lead to an $18 \%$ improvement in current density from $4.9 \mathrm{~mA} \mathrm{~cm}^{-2}$ for as-grown sample to $5.8 \mathrm{~mA} \mathrm{~cm}^{-2}$. We assign this enhanced performance to the reduced density of inherent point defects that was formed at the quantum dot (QD) and GaAs barrier. Post-growth thermal anneal treatment of QDSCs is demonstrated as a simple route for achieving improved device performance.
\end{abstract}




\section{Introduction}

Self-assembled quantum dots (QDs) have attracted much attention due to the interest in the fundamental physics of 3-dimensional confinement, which produces novel optoelectronic devices such as lasers, optical modulators, solar cells and mid-infrared photodetectors [1]. One of the indispensable features of using QD is the flexibility in tuning the effective band-gap, which allows us to tune the optical properties to correspond to the desired wavelengths. QDs are, thus desirably used to absorb specific parts of the solar spectrum within solar cells. In addition, they exhibit radiation tolerance as compared to their bulk counterpart, making them more suitable for space applications [2], [3]. More importantly, semiconductor QD solar cells (QDSCs) have the prerequisites for several proposed concepts for third generation photovoltaic, such as hot carrier[4], multi-exciton generation[5], and especially intermediate band solar cells (IBSC) [6], [7] which has an overwhelming theoretical efficiency of $63 \%$.

Although the proof-of-concept of QD-based IBSC has been demonstrated via two-photon absorption measurement [8], [9], [10], the intermediate band (IB) to conduction band (CB) absorption remains elusive at room temperature. This is due to several problems: (1) thermal escape of carriers obstructs the second photon absorption process [11] and (2) strain-induced defects from lattice mismatched heterostructures which form non-radiative (NR) recombination centres [12]. To date, several reports have demonstrated the use of high band gap barrier materials to reduce carrier thermal escape, including AlGaAs [8], [13] and InGaP [14]. More recently, direct QD doping has indicated partial thermal decoupling [15]. However, the techniques mentioned above involves the use of latticemismatch materials and introducing impurities, which could add defects to the active layer of the solar cell (SC). Although strain compensation spacer layers have been used, it usually involves using different bandgap materials [16], [17]. Hence, a post growth technique that restores the crystal lattice from defects - fortifying the active region from NR recombination centres, is desirable. Rapid thermal annealing (RTA) is a technique that has been extended to post-growth treatment to enhance structural and optical quality of nanostructures via interdiffusion of constituent atoms [18]. Moreover, the intermixing effect modifies the shape of the nanostructure, making it an alternative technique for 
changing the interband transition energies of QDs [19], [20]. In this work, we report the improvement of InAs/GaAs QDSC performance via post-growth RTA treatment without compromising the QD structures.

\section{Method}

Figure 1 shows a schematic illustration of the QDSC structure. The InAs/GaAs QDSC sample was grown by solid-source molecular bean epitaxy (MBE) on an epi-ready $n^{+}-G a A s$ (100) substrate. The

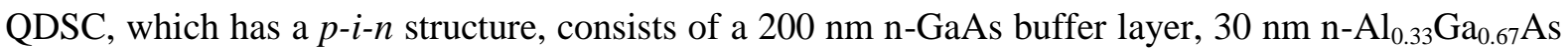
BSF, $300 \mathrm{~nm} \mathrm{n-GaAs} \mathrm{base,} \mathrm{20-layer} \mathrm{QD} \mathrm{structure,} 250 \mathrm{~nm}$-GaAs emitter, $30 \mathrm{~nm} \mathrm{p}-\mathrm{Al}_{0.75} \mathrm{Ga}_{0.25} \mathrm{As}$ window, and $250 \mathrm{~nm} \mathrm{p}^{+}$-GaAs contact layer. The QD layers consist of $2.5 \mathrm{ML}$ of InAs capped by 15 nm of GaAs, which separates the QD layers. The high growth temperature spacer layer technique, discussed in the earlier work[21], was used in the growth of these QDSCs. The sample was cleaved to equal sizes $\left(\sim 1 \mathrm{~cm}^{2}\right)$ followed by $\mathrm{SiO}_{2}$ coating $(\sim 180 \mathrm{~nm})$ using STS plasma-enhanced CVD at lowfrequency mode. RTA treatment of the samples was carried out using Solaris 150 RTP System at 650, 700 and $750{ }^{\circ} \mathrm{C}$. The annealing duration was 30 seconds with ramp-up rate $25^{\circ} \mathrm{C} / \mathrm{sec}$ under $\mathrm{N}_{2}$ ambient environment. The $\mathrm{SiO}_{2}$ coat was removed by dipping the samples into buffered HF bath.

Photoluminescence (PL) measurement was carried out at room temperature with excitation power of $18 \mathrm{~mW}$. The dispersed emission was collected through a lens system and directed to a monochromator with lock-in amplifier. The signal was picked up by a TE-cooled germanium detector. Temperaturedependent measurement consists of the same procedure with the sample mounted inside a cryostat subjected to circulating liquid helium.

SC devices were fabricated by thermally depositing Ni-AuGe-Ni-Au (5, 50, 25, $200 \mathrm{~nm})$ and AuZn alloy $(120 \mathrm{~nm})$ as the back and front (mask-patterned) contacts, respectively. No antireflection coating was deposited onto these SC devices. Current-density voltage (J-V) measurement was carried out with Keithley 2401 source meter with a temperature controlled stage (set to $25^{\circ} \mathrm{C}$ ) under a Xeon lamp which radiates collimated light with $100 \mathrm{~W} \mathrm{~cm}^{-2}$ which simulates AM $1.5 \mathrm{G}$ spectrum. External quantum efficiency (EQE) measurement was carried out with a SpeQuest Quantum Efficiency system. 


\section{Results and Discussion}

Figure 2a compares the PL spectra before and after RTA treatment. The as-grown sample shows a relative weak emission peak at $1080 \mathrm{~nm}$. A dramatic improvement of emission intensity of this peak is observed with RTA at 650 and $700{ }^{\circ} \mathrm{C}$, with negligible spectral shift. Further increase of the annealing temperature to $750{ }^{\circ} \mathrm{C}$ leads to a clear blue-shift of PL spectrum and a slight decrease in PL intensity, which is still stronger than that of the as-grown sample. Due to no obvious PL shift for the annealing at 650 and $700{ }^{\circ} \mathrm{C}$, the improvement in PL emission after RTA indicates greater efficiency of radiative recombination after annealing. This suggests that the pathway to NR recombination is supressed after annealing and can be linked to the removal of interior point defects in the active region, which is grown at a lower growth temperature. Although the QDs were grown with the high-growth temperature spacer layer technique at $580{ }^{\circ} \mathrm{C}$, part of the GaAs layers were grown at low temperatures and point defects, such as antisite defects, can still present in the low growth temperature materials due to low surface mobility of Ga adatoms and the excess of As [22]. This result where postannealing treatment improves photoemission is similar to those reported in references with improved PL after RTA, suggesting post-growth annealing can significantly improve the material quality by reducing the concentration of point defects [22] and [23]. The shoulder of the QD peak at $1040 \mathrm{~nm}$ is confirmed to be the first excited state (ES1) after power-dependent excitation shows saturation of the ground state (GS) peak. The full-width half-maximum (FWHM) and peak energy of the QDSCs annealed at $650^{\circ} \mathrm{C}$ and $700{ }^{\circ} \mathrm{C}$, is similar to that of the as-grown sample (see inset of Figure 2a), which indicates that no significant changes to the homogeneity and size of the dots have occurred. At $750{ }^{\circ} \mathrm{C}$, the significant blue shift, accompanied by decreased photoemission, indicates that the QDs have undergone compositional changes [24], [25], [26]. The blue shift of the PL spectrum can be explained by the intermixing of materials at higher temperatures leading to a compositional changes [23]. This is accompanied by a subsequent decrease in GS to ES1 transition from 46 to $39 \mathrm{meV}$ due to a shallower confinement. The decreased photoemission can also be explained by the reduced quantum confinement in the QDs, and thus increased rate of thermal escape, caused by thermally induced intermixing. On the other hand, the degradation of material quality upon high temperature annealing 
cannot be ruled out. Temperature-dependant PL was used to further study the effect of RTA on QDSCs. Figure $2 \mathrm{~b}$ plots the integrated PL intensity of the samples with and without RTA treatment. For all annealed samples, the integrated PL intensity is stronger than the as-grown sample at all measured temperatures, which confirms the recovery of material quality upon annealing. However, the lower integrated PL intensity of the sample annealed at $750{ }^{\circ} \mathrm{C}$ is observed at all temperatures compared to those annealed at 650 and $700{ }^{\circ} \mathrm{C}$. The reduced PL intensity for the sample annealed at $750{ }^{\circ} \mathrm{C}$ can thus be attributed to a higher NR recombination rate because the thermal escape of carriers is suppressed at low temperatures.

The J-V characteristics of the solar cells are shown in Fig. 3. After thermal annealing at $650{ }^{\circ} \mathrm{C}$, a distinct increase in short-circuit current is observed. Annealing the QDSC at $700^{\circ} \mathrm{C}$ exhibited a further increased short-circuit current density $\left(\mathrm{J}_{\mathrm{sc}}\right)$ from 4.9 to $5.8 \mathrm{mAcm}^{-2}$, which marks an $18 \%$ improvement from the reference cell. As the annealing temperature increases to $750{ }^{\circ} \mathrm{C}$, the shortcircuit current is still higher than the as-grown SC but lower than the ones annealed at 650 and $700{ }^{\circ} \mathrm{C}$. Additionally, the change of open circuit voltage $\left(\mathrm{V}_{\mathrm{oc}}\right)$ upon annealing follows the same trend as shortcircuit current. The inset of Figure 3 shows the change in $\mathrm{J}_{\mathrm{sc}}$ and $\mathrm{V}_{\mathrm{oc}}$ with annealing temperature. The QDSC reference cell has a $\mathrm{V}_{\mathrm{oc}}$ of $0.79 \mathrm{~V}$, this is consistent with the value previously reported in [15] and [27]. RTA at $700{ }^{\circ} \mathrm{C}$ demonstrates an increased $\mathrm{V}_{\text {oc }}$ by $30 \mathrm{mV}$ to $0.82 \mathrm{~V}$. This correlates well with the PL studies that suggest RTA assists the removal of defects. By improving in the crystal quality of the active medium, there should be reduced impediment to the mobility of the carriers. Thus, carriers have a longer diffusion length, which gives a greater probability of charge extraction to occur before the entering the recombination pathway. Hence, the reduced NR recombination allows a higher voltage to be achieved by the SC. Furthermore, the RTA might also reduce defect assisted tunnelling out of the QDs which could improve the open circuit voltage as observed[28], [29]. However, annealing at $750{ }^{\circ} \mathrm{C}$ causes both $\mathrm{J}_{\mathrm{sc}}$ and $\mathrm{V}_{\mathrm{oc}}$ to decrease leading to a reduction in SC performance. This can be attributed to the presence of excessive Ga vacancies in the GaAs diffused from the $\mathrm{GaAs} / \mathrm{SiO}_{2}$ interface at elevated annealing temperature [30]. This degradation of material quality at the annealing 
temperature of $750{ }^{\circ} \mathrm{C}$ is supported by the reduced photoemission intensity observed in the PL measurement for QDSCs annealed at $750{ }^{\circ} \mathrm{C}$.

To gain further insight into the annealing effects on the QDSC, the EQE spectra of QDSCs with and without post-growth annealing are compared in Fig. 4. Figure 4 shows an increase in spectral response between $450-900 \mathrm{~nm}$ after RTA treatments, which reveals that the improvement in $\mathbf{J}_{\mathrm{sc}}$ can largely be attributed to the GaAs layers of the QDSC. This provides further evidence to support the removal of defects from the GaAs region via RTA. The inset of Figure 4 shows a magnified view from the spectral region that corresponds to QD absorption, which shows an increased spectral response from QDs after annealing when compared to the un-annealed QDSC. The greatest spectral response was achieved after RTA at $700{ }^{\circ} \mathrm{C}$, but at $750{ }^{\circ} \mathrm{C}$ the response reduces which indicates too high temperature annealing can degrade material quality [31]. This correlates well with the results exhibited in the $\mathrm{J}-\mathrm{V}$ measurement. The EQE gives evidence to suggest the reduction of $\mathrm{J}_{\mathrm{sc}}$ at $750{ }^{\circ} \mathrm{C}$ is primarily due to the GaAs region, as the spectral response from the QD region is almost unchanged. This signifies that RTA can remove point defects from GaAs grown at $580{ }^{\circ} \mathrm{C}$, but at annealing temperatures $\left(\geq 750^{\circ}\right)$ vacancy or interstitial defects may re-generate, which distributes in the GaAs region [30].

We have demonstrated that QDSCs annealed at temperatures above $750{ }^{\circ} \mathrm{C}$ begin to reduce the QD photoemission peak, or merge with that of wetting layer. We believe that above this temperature the QDs begin to dissolve into the wetting layer forming quantum well structures and lose the optical/electronic properties exhibited by QDs, including 3-dimensional confinement. Hence, the upper limit for RTA of QDSCs is $750{ }^{\circ} \mathrm{C}$ and in order to sustain the prerequisites for IBSCs, RTA should be kept below this value. Previous reports have shown that intermixing upon RTA processing has facilitated tuning to the desired spectral response[20], [25], [32]. In our studies, substantial tuning is initiated when the annealing temperature is above $700{ }^{\circ} \mathrm{C}$.

\section{Conclusion}


Conventional InAs/GaAs QDSC grown in SK mode has been subjected to post-growth annealing. The performance of QDSCs has improved with annealing, achieving the highest $\mathrm{J}_{\mathrm{sc}}\left(5.8 \mathrm{~mA} \mathrm{~cm}{ }^{-2}\right)$ and $\mathrm{V}_{\mathrm{oc}}$ $(0.82 \mathrm{~V})$ after annealing at $700{ }^{\circ} \mathrm{C}$ for 30 seconds. PL measurements confirmed the presence of QDs after annealing, up to $750{ }^{\circ} \mathrm{C}$. From the enhanced photoemission and improved spectral response, we can deduce that RTA has facilitated the restoration of crystal quality in both the InAs and GaAs material in the active layer. This is achieved by reducing the density of point defects in the low growth temperature GaAs spacer layers.

\section{Acknowledgement}

The authors acknowledge the Royal Society, the Defense Science Technology Laboratory and UK Engineering and Physics Research Council (EPSRC) through Grant EP/K029118/1 for funding support. 


\section{Reference}

[1] H. C. Liu, M. Gao, J. McCaffrey., et al.: 'Quantum dot infrared photodetectors', Applied Physics Letters, 2001, 78, (1), pp 79-81

[2] C. Kerestes, D. V. Forbes, Z. Bittner., et al.: 'Strain Effects on Radiation Tolerance of Quantum Dot Solar Cells', IEEE journal of Photovoltaics, 2011, 4, (1), pp 224-232

[3] C. D. Cress, S. M. Hubbard, B. J. Landi., et al.: 'Quantum dot solar cell tolerance to alpha-particle irradiation', Applied Physics Letters, 2007, 91, pp 183108

[4] R. T. Ross, A. J. Nozik: 'Efficiency of hot-carrier solar energy converters', Journal of Applied Physics, 1982, 53, (5), pp 3813

[5] A. J. Nozik: 'Exciton multiplication and relaxation dynamics in quantum dots: applications to ultrahigh-efficiency solar photon conversion', Inorganic chemistry, 2005, 44, (20), pp 6893-9

[6] A. Luque and A. Mart1, 'Increasing the Efficiency of Ideal Solar Cells by Photon Induced Transitions at Intermediate Levels', Physical Review Letters, 1997, 78, (26), pp 5014-5017

[7] A. Luque and A. Marti, 'The intermediate band solar cell: progress toward the realization of an attractive concept', Advanced Materials, 2010, 22, (2), pp 160-174

[8] N. Ahsan, N. Miyashita, M. Monirul Islam., et al.: "Two-photon excitation in an intermediate band solar cell structure," Applied Physics Letters, 2012, 100, (17), pp 172111

[9] T. Sogabe, Y. Shoji, M. Ohba., et al.: 'Intermediate-band dynamics of quantum dots solar cell in concentrator photovoltaic modules', Scientific reports, 2014, 4, pp 4792

[10] A. Martí, E. Antolín, C. Stanley, et al.: 'Production of Photocurrent due to Intermediate-to-Conduction-Band Transitions: A Demonstration of a Key Operating Principle of the Intermediate-Band Solar Cell', Physical Review Letters, 2006, 97, (24) pp 247701

[11] P. G. Linares, A. Martí, E. Antolín, et al.: 'Voltage recovery in intermediate band solar cells', Solar Energy Materials and Solar Cells, 2012, 98, pp 240-244

[12] C. Himwas, S. Panyakeow, and S. Kanjanachuchai, 'Optical properties of as-grown and annealed InAs quantum dots on InGaAs cross-hatch patterns', Nanoscale research letters, 2011, 6, (1), pp 496

[13] K. A. Sablon, J. W. Little, V. Mitin, et al.: 'Strong Enhancement of Solar Cell Efficiency Due to Quantum Dots', Nano letters, 2011, 11, pp 2311-2317

[14] D. V. Forbes, A. M. Podell, M. A. Slocum, et al.: 'OMVPE of InAs quantum dots on an InGaP surface', Materials Science in Semiconductor Processing, 2013, 16, (4), pp $1148-1153$ 
[15] P. Lam, S. Hatch, J. Wu, et al.: "Voltage recovery in charged InAs/GaAs quantum dot solar cells," Nano Energy, 2014, 6, pp 159-166

[16] R. B. Laghumavarapu, M. El-Emawy, N. Nuntawong, et al.: 'Improved device performance of InAs/GaAs quantum dot solar cells with $\mathrm{GaP}$ strain compensation layers', Applied Physics Letters, 2007, 91, (24), pp 243115

[17] Y. Okada, T. Morioka, K. Yoshida, et al.: 'Increase in photocurrent by optical transitions via intermediate quantum states in direct-doped InAs/GaNAs straincompensated quantum dot solar cell', Journal of Applied Physics, 2011, 109, (2), pp 024301

[18] A. Babiński, J. Jasiński, R. Bożek, et al.: 'Rapid thermal annealing of InAs/GaAs quantum dots under a GaAs proximity cap', Applied Physics Letters, 2011, 79, (16), pp 2576

[19] R. Gunawan, M. Y. L. Jung, E. G. Seebauer, et al.: 'Optimal control of rapid thermal annealing in a semiconductor process', Journal of Process Control, 2004, 14, (4), pp $423-430$

[20] W. H. Ng, E. a. Zibik, L. R. Wilson, et al.: 'Tuning of intraband absorption and photoresponse in self-assembled InAs/GaAs quantum dots by thermal annealing', Journal of Applied Physics, 2008, 103, (6), pp 066101

[21] F. K. Tutu, I. R. Sellers, M. G. Peinado, et al.: 'Improved performance of multilayer InAs/GaAs quantum-dot solar cells using a high-growth-temperature GaAs spacer layer', Journal of Applied Physics, 2012, 111, (4), pp 046101

[22] Z. Liliental-Weber, X. W. Lin, J. Washburn, et al.: 'Rapid thermal annealing of lowtemperature GaAs layers,' Applied Physics Letters, 1995, 66, (16), pp 2086

[23] J. Guo-Zhi, Y. Jiang-hong, S. Yong-chun, et al.: 'Evolution of InAs Quantum Dots during Annealing Process', Acta Physica Polonica A, 2008, 114, (4), pp 919-923

[24] X. Zhou, Y. Chen, and B. Xu, 'Optical identification of electronic state levels of an asymmetric InAs/InGaAs/GaAs dot-in-well structure' Nanoscale research letters, 2011, 6, (1), pp 317

[25] L. Fu, P. Kuffner, I. McKerracher, et al.: 'Rapid thermal annealing study of InGaAs/GaAs quantum dot infrared photodetectors grown by metal-organic chemical vapor deposition," Lasers and Electro-Optics Society, 2005. The 18th Annual Meeting of the IEEE, Oct. 2005, pp 228-229

[26] K. Stewart, M. Buda, L. Fu, et al.: 'Strain relaxation in rapid thermally annealed InAs/GaAs quantum dot infrared photodetectors', Optoelectronic and Microelectronic Materials and Devices, 2002 Conference of IEEE, Dec 2002, pp 475-478

[27] S. Hatch, J. Wu, K. Sablon, et al.: 'InAs/GaAsSb quantum dot solar cells', Optics Express, 2014, 22, (S3), pp A679 
[28] O. Breitenstein, J. Bauer, A. Lotnyk, et al.: 'Defect induced non-ideal dark characteristics of solar cells', Superlattices and Microstructures, 2009, 45, (4-5), pp 182-189

[29] A. Kaminski, J. J. Marchand, and A. Laugier, "Non ideal dark I - V curves behavior of silicon solar cells," Solar Energy Materials and Solar Cells, 1998, 51, pp 221-231

[30] Z. Y. Zhang, Q. Jiang, I. J. Luxmoore, et al.: 'A p-type-doped quantum dot superluminescent LED with broadband and flat-topped emission spectra obtained by post-growth intermixing under a GaAs proximity cap', Nanotechnology, 2009, 20, (5), pp 055204

[31] S. J. Xu, X. C. Wang, S. J. Chua, et al.: 'Effects of rapid thermal annealing on structure and luminescence of self-assembled InAs/GaAs quantum dots', Applied Physics Letters, 1998, 72, (25), pp 3335

[32] K. Stewart, M. Buda, J. Wong-Leung, et al.: 'Influence of rapid thermal annealing on a 30 stack InAs/GaAs quantum dot infrared photodetector', Journal of Applied Physics, 2003, 94, (8), pp 5283 


\section{Figures}

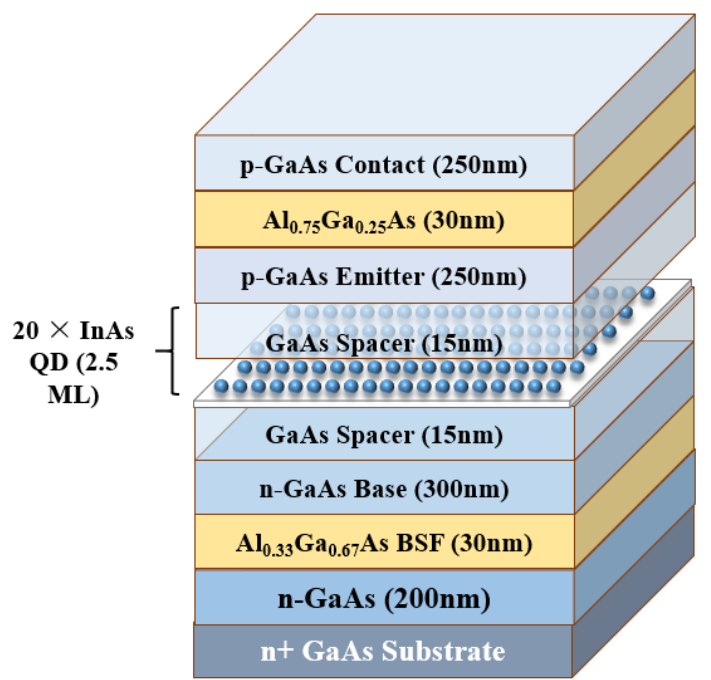

Figure 1: Schematic illustration of the quantum dot solar cell structure, grown with 20 repeats of InAs QD layers, each separated by $15 \mathrm{~nm}$ of GaAs spacer.
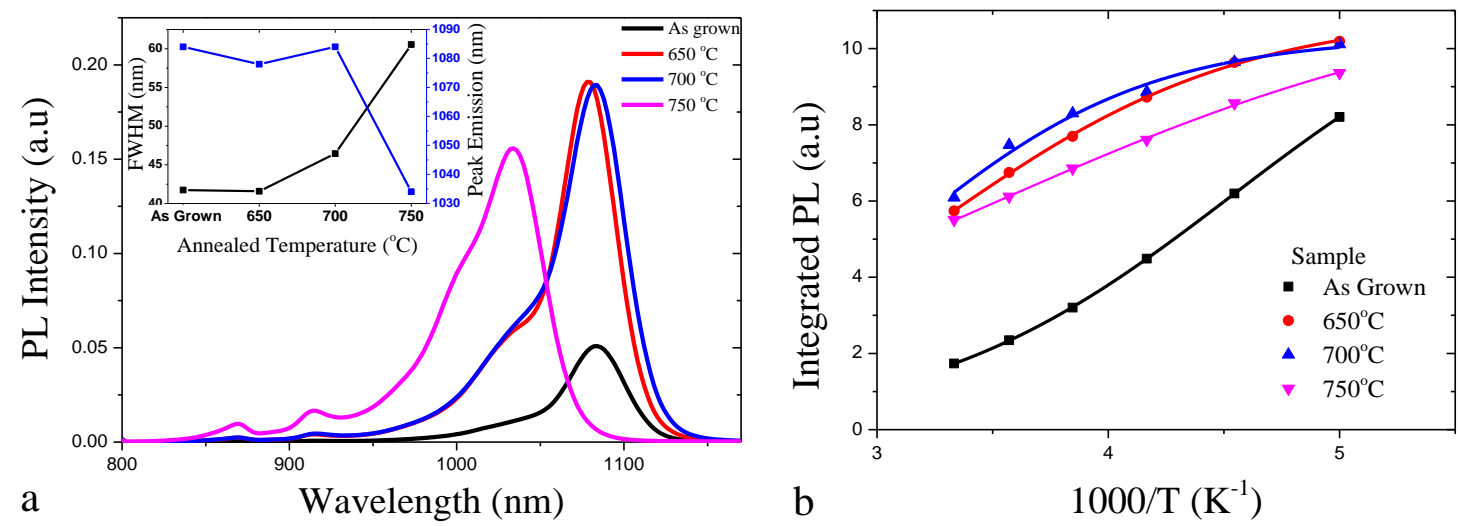

Figure 2: (a) Photoluminescence measurement shows the emission of QD peak with excitation power of $18 \mathrm{~mW}$, which corresponds to $\sim 1080 \mathrm{~nm}$ for as-grown QDSC at room temperature. An increase in photoemission is observed after the subjection to rapid thermal annealing. The inset shows the change of full width of half maximum and peak emission as annealing temperature increases. The shoulder of the ground state emission peak is confirmed to be a first excited state via power-dependent 
photoluminescence. The increase in photoemission indicates fewer NR recombination centres. (b) Integrated PL intensity as a function of temperature.

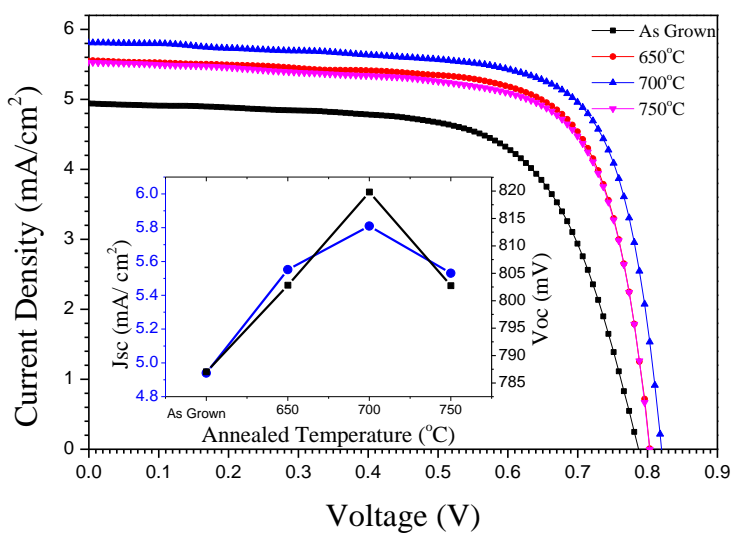

Figure 3: J-V curves showing the solar cell performance under AM $1.5 \mathrm{G} 1$ sun $\left(1000 \mathrm{~W} \mathrm{~m}^{-2}\right)$ illumination. The slight increase in open-circuit voltage is accompanied by an improvement in current density. The inset of the graph shows the change in short-circuit current density and open-circuit voltage after annealing. At annealing temperature $700^{\circ} \mathrm{C}$, an improvement in current density (14\%) and open-circuit voltage (4\%) as compared to the as-grown sample is seen.

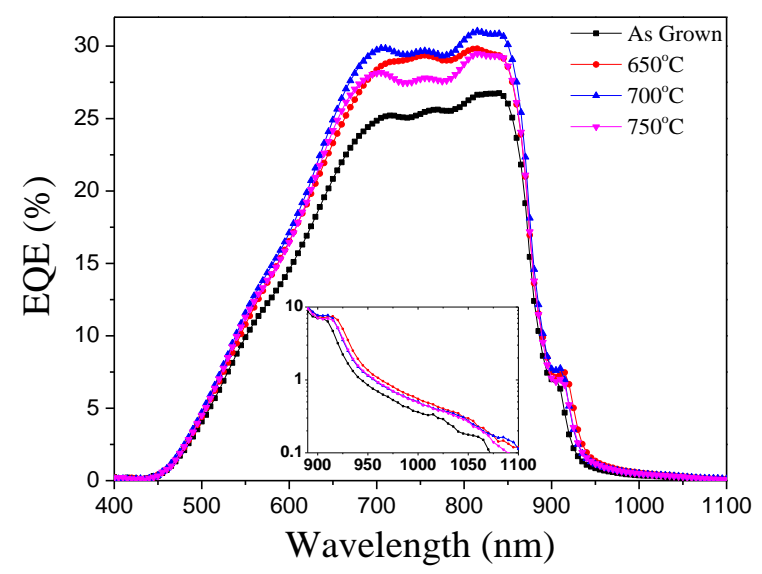

Figure 4: External quantum efficiency measured at $25^{\circ} \mathrm{C}$ shows an improvement in the spectral response for the wavelength range corresponding to GaAs. The inset shows an enlarged view of the spectral region corresponding to the QDs. 